\title{
Uric acid and cardiometabolic risk profile in youths with type 1 diabetes: sex-related associations
}

Procolo Di Bonito

"S. Maria delle Grazie", Pozzuoli Hospital

\section{Francesco Maria Rosanio}

University of Naples Federico II

Maria Loredana Marcovecchio

University of Cambridge

Valentino Cherubini

Fondazione Ospedale Salesi

Maurizio Delvecchio

Azienda Universitaria Ospedaliera Consorziale - Policlinico Bari

Francesca Di Candia

University of Naples Federico II

Dario lafusco

University of Campania "Luigi Vanvitelli"

\section{Angela Zanfardino}

University of Campania "Luigi Vanvitelli"

Brunella lovane

University of Parma

Claudio Maffeis

University of Verona

Giulio Maltoni

University of Bologna

Carlo Ripoli

Azienda Ospedaliera G. Brotzu

\section{Elvira Piccinno}

Azienda Universitaria Ospedaliera Consorziale - Policlinico Bari

Claudia Anita Piona

University of Verona

Maria Rossella Ricciardi

Azienda Ospedaliera G. Brotzu

Riccardo Schiaffini

Bambino Gesù Children's Hospital 
University of Naples Federico II

Enza Mozzillo ( $\nabla$ enza.mozzillo@unina.it )

University of Naples Federico II

\section{Research Article}

Keywords: type 1 diabetes, children, glomerular filtration rate, uric acid, cardio metabolic risk

Posted Date: February 14th, 2022

DOI: https://doi.org/10.21203/rs.3.rs-1295358/v1

License: (c) (1) This work is licensed under a Creative Commons Attribution 4.0 International License. Read Full License 


\section{Abstract}

The aim of this study was to investigate the association between uric acid (UA) and cardiometabolic risk factors (CMRFs) by sex in youths with type 1 diabetes (T1D). Retrospective data collected from 1323 youths (5-18 years; 716 boys) with T1D recruited in 9 Italian Pediatric Diabetes Centers were analyzed. CMRFs included $\mathrm{UA}, \mathrm{HbA}_{1 \mathrm{c}}$, blood pressure (BP), cholesterol (TC), $\mathrm{HDL}$, triglycerides (TG), neutrophils (N) and lymphocytes (L) count, glomerular filtration rate (eGFR) (calculated using Schwartz-Lyon equation). The distribution of CMRFs was analyzed in sex-specific tertiles of UA. In the whole study population, statistically significant differences were found across $\mathrm{UA}$ tertiles for age, $\mathrm{BMI}$, daily insulin dose, $\mathrm{HbA}_{1 \mathrm{c}}$, HDL, TG, TG/HDL ratio, TC/HDL ratio, SPISE index, N/L ratio, systolic BP, eGFR ( $p=0.01-0.0001)$. The highest tertile of UA was associated with 1,5-3,6 fold increased risk of high TG/HDL ratio, TC/HDL ratio, high BP, and MRGFR, compared to the lowest tertile in boys. Instead, in girls the highest tertile of UA was associated with two-fold increased risk of TC/HDL ratio, N/L ratio and MRGFR. Uric acid may represent a useful marker for identifying youth with T1D at high cardiometabolic risk, and association appears to vary by sex.

\section{Introduction}

Several cross-sectional and longitudinal studies have reported the independent role of uric acid (UA) as a predictor of cardiovascular morbidity, mortality and incident chronic kidney disease (CKD), in adult populations, especially with type 2 diabetes [1-4]. A robust association between UA and risk of incident cardiovascular events, mortality and an early onset of GFR decline has also been reported in adults with type 1 diabetes (T1D) [5-7]. The mechanisms linking UA to cardio-renal disease risk are not fully elucidated yet. However, the effect of UA might be mediated by the activation of oxygen free radicals which in turn increase oxidative stress and inflammation [1, 8].

Several cross-sectional studies in pediatric populations, primarily performed in obese children, demonstrated that high UA levels are associated to metabolic syndrome (MetS) and its individual components, nonalcoholic fatty liver disease, reduced estimated glomerular filtration rate (eGFR) and glucose dysmetabolism [9-11].

In contrast, in adolescents with T1D, the relationship between UA levels and cardiometabolic risk factors (CMRFs) is controversial and little explored $[12,13]$. This is an important issue which deserves clarification since it is well known that the cardiovascular prevention should start as early as possible, not only in children with obesity and type 2 diabetes but also in those with T1D [14-16]. Furthermore, a potential sex-related difference in the relationship between UA and CMRF has been not well explored so far $[12,13]$ Therefore, the aim of the present study was to evaluate the CMR profile associated with UA levels in a large sample of children and adolescents with T1D. In addition we assessed whether the UArelated CMR profile is different among the two sexes.

\section{Methods}




\section{Measurements}

For all study participants, the following data were recorded by each of the nine participating centers: age, sex, height, weight, body mass index (BMI), blood pressure (BP), serum creatinine (Scr), UA, $\mathrm{HbA}_{1 \mathrm{c}}$, total cholesterol (TC), triglycerides (TG), HDL, Neutrophil (N) and Lymphocyte (L) counts, albuminuria and insulin regimen (multiple daily injections vs continuous subcutaneous insulin infusion) and doses (IU/Kg/day). BMI-SDS was calculated based on the Italian BMI normative data [30].

In all centers $\mathrm{HbA}_{1 \mathrm{c}}$ was assessed by High-Performance Liquid Chromatography; TC, TG and HDL were determined by enzymatic methods; $\mathrm{N}$ and $\mathrm{L}$ counts were measured using an automated analyzer.

Scr was analyzed in 4 centers by enzymatic method and in 5 centers using Jaffé IDMS traceable method. The relationship between Scr, age, sex, BMI and enzymatic method was explored by a linear regression analysis to harmonize the Scr levels assessed by Jaffè method and enzymatic method as elsewhere described [21].

The following parameters: TG to HDL (TG/HDL), TC to HDL (TC/HDL) and Neutrophil to Lymphocyte ratio $(\mathrm{N} / \mathrm{L})$ were calculated. The single-point insulin sensitivity estimator (SPISE index), a surrogate index of insulin-sensitivity, was calculated with the following formula:

$600 \times\left(H D L^{0.185}\right) /\left(T^{0.2} \times B M I^{1.338}\right)[24]$.

Estimated glomerular filtration rate (eGFR) was calculated using Schwartz-Lyon equation:

eGFR $=[k x$ height $(\mathrm{cm}) / \mathrm{Scr}(\mathrm{mg} / \mathrm{dL})], k=36.5$ in males aged $>13$ years, $k=32.5$ in others [31].

Evaluation of albuminuria was determined in 4 centers $(n=697)$ as albumin to creatinine ratio (ACR) (using an enzymatic method for assessment of Scr) on first-morning urine samples, while in 5 centers ( $\mathrm{n}=$ 626) as albumin excretion rate (AER) in 24 hours urine samples, as recommended by the kidney disease: Improving Global Outcomes (KDIGO) clinical practice guideline [32].

BP was measured following the recommendations by the European Society of Hypertension [33]. BP was measured, after 5 min of resting in a quiet room, using an appropriate sized arm cuff on the right arm and an aneroid sphygmomanometer. Three measurements were obtained every $2 \mathrm{~min}$ and the mean of the last two values were used in the analyses.

All blood samples for biochemical analyses were collected in each center after $12 \mathrm{~h}$ of fasting. Although laboratory analyses were performed in different laboratories, these are all part of the Italian National Health System and are certified according to International Standards ISO 9000 (www.iso9000.it/) [29].

\section{Definitions}

Autoimmune diseases were defined by the presence of autoimmune thyroiditis and/or celiac disease. Albuminuria (microalbuminuria) was defined as moderately increased (ACR 30-300 mg/g; AER 30-300 
$\mathrm{mg} / \mathrm{d}$ ) or severely increased (ACR $>300 \mathrm{mg} / \mathrm{g},>300 \mathrm{mg} / \mathrm{d}$ ) (macroalbuminuria) [32]. The presence of albuminuria was defined by positivity in at least two different measurements [34].

High BP was defined using criteria proposed by the American Academy of Pediatrics: BP $\geq 95^{\text {th }}$ percentile for age, sex and height in children aged less than 13 years or $B P \geq 130 / 80 \mathrm{mmHg}$ in adolescents (age $\geq 13$ years) [35].

High TG/HDL ratio, TC/HDL ratio and N/L ratio were defined by the $75^{\text {th }}$ percentile of the study population. Mildly reduced eGFR was defined by a value between $60-89 \mathrm{~mL} / \mathrm{min} / 1.73 \mathrm{~m}^{2}$ [32].

\section{Statistics}

Data are expressed as mean \pm standard deviation, median and interquartile range, or absolute and relative frequencies, unless otherwise stated. Variables with skewed distribution such as $\mathrm{HbA}_{1 \mathrm{c}}, \mathrm{TG}, \mathrm{TG} / \mathrm{HDL}$ ratio, $T C / H D L$ ratio, $N / L$ ratio and insulin dose were log-transformed for the statistical analysis and showed in the tables as median and interquartile range.

Between groups differences in continuous variables were assessed with Student's $t$-test. Analysis of variance (ANOVA) was applied to evaluate differences across tertiles of UA. The $\chi^{2}$ and Fisher's exact tests, as appropriate, were used to compare categorical variables. Exact tests were performed using the Monte Carlo method.

To evaluate the association (Odds ratio, $95 \% \mathrm{Cl}$ ) between the CMRFs and sex-specific tertiles of UA, a logistic regression analysis was performed adjusted for centers, age, insulin dose (IU/Kg/day), $\mathrm{HbA}_{1 \mathrm{c}}$ or BMI-SDS. Each single CMRF was included as a dependent variable and the sex-specific tertiles of UA (independent variables) were included as categorical covariates, applying a contrast between phenotypes and using the first tertile as the reference category.

Statistical analysis was performed using the IBM SPSS Statistics for Windows, Version 20.0. Armonk, NY. A $p$-value $<0.05$ was considered statistically significant.

\section{Results}

This multicenter retrospective cross-sectional study included Caucasian children and adolescents with T1D recruited consecutively recruited within 16 months from 1 January 2019 to 30 April 2020 in 9 Italian Pediatric Diabetes Centers that are part of the Diabetes Study Group of the Italian Society for Pediatric Endocrinology and Diabetology (ISPED).

Medical history, biochemical and clinical data were retrieved from clinical records. Inclusion criteria were: diagnosis of T1D (at least 1 positive autoantibody), diabetes duration $>1$ year, age 5-18 years, availability of anthropometric and biochemical data, including UA. 
The study population included 1323 patients with T1D, 716 boys and 607 girls, with a mean ( \pm SD) age of 13.5 \pm 3.1 . The clinical and biochemical characteristics of the study population are summarized in Table 1 . The overall sample was divided into sex-specific tertiles of UA and the characteristics of the sample are reported in Table 2. In the whole study population, statistically significant differences were found across UA tertiles for age, BMI, daily insulin dose, $\mathrm{HbA}_{1 c}, \mathrm{HDL}, \mathrm{TG}, \mathrm{TG} / \mathrm{HDL}$ ratio, TC/HDL ratio, SPISE index, N/L ratio, systolic BP, eGFR, across tertiles of UA ( $p=0.01-0.0001)$. 
Table 1

Anthropometric and biochemical features of the study population by sex.

\begin{tabular}{|c|c|c|c|c|}
\hline & All & Boys & Girls & $p$ value \\
\hline$n$ & 1323 & 716 & 607 & \\
\hline Age (years) & $13.5 \pm 3.1$ & $13.6 \pm 3.0$ & $13.4 \pm 3.2$ & 0.141 \\
\hline BMI $\left(\mathrm{kg} / \mathrm{m}^{2}\right)$ & $20.7 \pm 3.7$ & $20.7 \pm 3.7$ & $20.8 \pm 3.7$ & 0.618 \\
\hline BMI-SDS & $0.05 \pm 1.0$ & $-0.02 \pm 1.0$ & $0.13 \pm 1.0$ & 0.005 \\
\hline Diabetes duration (years) & $6.5 \pm 3.3$ & $6.5 \pm 3.2$ & $6.4 \pm 3.3$ & 0.531 \\
\hline Autoimmune diseases (\%) & $299(22.6)$ & $156(22)$ & $143(23.6)$ & 0.443 \\
\hline Insulin dose (IU/Kg/day) & $0.90(0.69-1.14)$ & $0.89(0.67-1.13)$ & $0.90(0.72-1.15)$ & 0.020 \\
\hline $\mathrm{HbA}_{1 \mathrm{c}}(\%)$ & $7.7(7.0-8.4)$ & $7.6(7.1-8.4)$ & $7.7(7.0-8.5)$ & 0.928 \\
\hline $\mathrm{HbA1c}(\mathrm{mmol} / \mathrm{mol})$ & $60.7(53.0-68.0)$ & $60.0(54.0-68.0)$ & $61.0(53.0-69.0)$ & 0.928 \\
\hline CSII, n (\%) & $460(35)$ & $245(34)$ & $215(35)$ & 0.647 \\
\hline Albuminuria, (\%) & $90(6.8)$ & $37(5.2)$ & $53(8.7)$ & 0.010 \\
\hline Total cholesterol (mg/dL) & $165.0 \pm 31.4$ & $165.5 \pm 31.7$ & $164.3 \pm 31.1$ & 0.511 \\
\hline $\mathrm{HDL}(\mathrm{mg} / \mathrm{dL})$ & $59.2 \pm 12.8$ & $59.5 \pm 12.4$ & $58.8 \pm 13.2$ & 0.372 \\
\hline Triglycerides (mg/dL) & $58.0(46.0-75.0)$ & $58.0(45.0-75.0)$ & $58.0(47.0-76.0)$ & 0.478 \\
\hline TG/HDL ratio & $1.02(0.74-1.39)$ & $1.00(0.73-1.37)$ & $1.04(0.75-1.42)$ & 0.301 \\
\hline TC/HDL ratio $(\mathrm{mg} / \mathrm{dL})$ & $2.8(2.4-3.2)$ & $2.8(2.4-3.3)$ & $2.7(2.4-3.3)$ & 0.508 \\
\hline SPISE index & $10.2 \pm 2.7$ & $10.3 \pm 2.7$ & $10.2 \pm 2.7$ & 0.315 \\
\hline $\mathrm{N} / \mathrm{L}$ ratio & $1.35(1.01-1.80)$ & $1.36(1.02-1.80)$ & $1.35(1.00-1.80)$ & 0.213 \\
\hline Systolic BP (mmHg) & $109.0 \pm 11.8$ & $109.3 \pm 12.1$ & $108.6 \pm 11.4$ & 0.268 \\
\hline Diastolic BP (mmHg) & $67.4 \pm 7.9$ & $67.3 \pm 8.1$ & $67.6 \pm 7.6$ & 0.458 \\
\hline eGFR (mL/min/1.73m²) & $103.7 \pm 26.0$ & $106.1 \pm 25.9$ & $100.9 \pm 25.9$ & $<0.0001$ \\
\hline Uric acid (mg/dL) & $3.50 \pm 1.0$ & $3.53 \pm 1.0$ & $3.47 \pm 1.0$ & 0.274 \\
\hline \multicolumn{5}{|c|}{$\begin{array}{l}\text { Data are expressed as mean } \pm \mathrm{SD}, \mathrm{n}(\%), \text { median (interquartile range). } P \text { values are for differences } \\
\text { between boys and girls. Abbreviations: } \mathrm{HbA}_{1} \text { : glycosylated hemoglobin, } \mathrm{CSIl} \text { : continuous } \\
\text { subcutaneous insulin infusion. } \mathrm{MDI} \text { : multiple doses of insulin. IU/Kg/day: insulin dose pro Kg Day. } \\
\text { TG/HDL ratio: triglycerides to } \mathrm{HDL} \text { ratio. TC/HDL ratio: Cholesterol to HDL ratio. SPISE: single-point } \\
\text { insulin sensitivity estimator } \mathrm{N} / \mathrm{L} \text { ratio: Neutrophil/Lymphocyte ratio. } \mathrm{BP}: \text { blood pressure. eGFR: } \\
\text { estimated glomerular filtration rate }\end{array}$} \\
\hline
\end{tabular}


Table 2

Anthropometric and biochemical features of the study population according to sex-specific tertiles of uric acid.

\begin{tabular}{|c|c|c|c|c|}
\hline & Uric acid tertiles & & & \\
\hline \multirow[t]{2}{*}{$\mathrm{UA}(\mathrm{mg} / \mathrm{dl})$} & $<3.0$ in boys & $\geq 3.0<3.9$ in boys & $\geq 3.9$ in boys & \\
\hline & $<3.0$ in girls & $\geq 3.0<3.8$ in girls & $\geq 3.8$ in girls & \\
\hline$n=1323$ & 403 & 471 & 449 & $p$ value \\
\hline Age (years) & $13.1 \pm 3.1$ & $13.6 \pm 3.1$ & $13.8 \pm 3.0$ & 0.001 \\
\hline Boys, n (\%) & $216(54)$ & $254(54)$ & $246(55)$ & 0.936 \\
\hline $\mathrm{BMI}\left(\mathrm{kg} / \mathrm{m}^{2}\right)$ & $20.2 \pm 3.5$ & $20.7 \pm 3.8$ & $21.1 \pm 3.9$ & 0.001 \\
\hline BMI-SDS & $-0.02 \pm 0.9$ & $0.01 \pm 1.0$ & $0.10 \pm 1.0$ & 0.206 \\
\hline Diabetes duration (years) & $6.3 \pm 3.2$ & $6.6 \pm 3.3$ & $6.5 \pm 3.3$ & 0.306 \\
\hline Autoimmune diseases & $85(21)$ & $99(21)$ & $115(26)$ & 0.168 \\
\hline Insulin dose (IU/Kg/day) & $0.85(0.66-1.06)$ & $0.87(0.66-1.13)$ & $0.96(0.75-1.21)$ & 0.001 \\
\hline $\mathrm{HbA}_{1 \mathrm{c}}(\%)$ & $7.7(7.2-8.5)$ & $7.6(7.1-8.4)$ & $7.6(7.0-8.3)$ & 0.028 \\
\hline $\mathrm{HbA}_{1 \mathrm{c}}(\mathrm{mmol} / \mathrm{mol})$ & $61.0(55.0-69.4)$ & $60.0(54.0-68.0)$ & $60.0(53.0-67.2)$ & 0.028 \\
\hline CSII, n (\%) & $141(35)$ & $172(36)$ & $147(33)$ & 0.482 \\
\hline Albuminuria, (\%) & $25(6.2)$ & $34(7.2)$ & $31(6.9)$ & 0.834 \\
\hline Total cholesterol (mg/dL) & $166.1 \pm 32.0$ & $166.6 \pm 31.5$ & $162.2 \pm 30.5$ & 0.073 \\
\hline $\mathrm{HDL}(\mathrm{mg} / \mathrm{dL})$ & $61.5 \pm 12.8$ & $59.1 \pm 12.8$ & $57.2 \pm 12.4$ & $<0.0001$ \\
\hline Triglycerides $(\mathrm{mg} / \mathrm{dL})$ & $56.0(45.0-70.0)$ & $59.0(47.0-75.0)$ & $60.0(47.0-85.0)$ & 0.001 \\
\hline TG/HDL ratio & $0.93(0.68-1.22)$ & $1.04(0.74-1.42)$ & $1.07(0.78-1.51)$ & $<0.0001$ \\
\hline $\mathrm{TC} / \mathrm{HDL}$ ratio $(\mathrm{mg} / \mathrm{dL})$ & $2.6(2.3-3.1)$ & $2.8(2.4-3.3)$ & $2.8(2.5-3.3)$ & 0.008 \\
\hline SPISE index & $10.7 \pm 2.8$ & $10.2 \pm 2.6$ & $9.8 \pm 2.7$ & $<0.0001$ \\
\hline $\mathrm{N} / \mathrm{L}$ ratio & $1.32(0.97-1.69)$ & $1.33(1.01-1.74)$ & $1.40(1.08-1.93)$ & 0.001 \\
\hline
\end{tabular}

Data are expressed as mean \pm SD, $\mathrm{n}(\%)$, median (interquartile range). $P$ values are for trends across tertiles.

Abbreviations: $\mathrm{HbA}_{1 \mathrm{c}}$ : glycosylated hemoglobin, CSII: continuous subcutaneous insulin infusion. MDI: multiple doses of insulin. IU/ $\mathrm{Kg} /$ day: insulin dose pro Kg Day. TG/HDL ratio: triglycerides to HDL ratio. TC/HDL ratio: Cholesterol to HDL ratio. SPISE: single-point insulin sensitivity estimator N/L ratio: Neutrophil/Lymphocyte ratio. BP: blood pressure. eGFR: estimated glomerular filtration rate 


\begin{tabular}{|c|c|c|c|c|}
\hline \multicolumn{5}{|c|}{ Uric acid tertiles } \\
\hline Systolic BP (mmHg) & $107.2 \pm 11.5$ & $108.8 \pm 12.1$ & $110.6 \pm 11.4$ & $<0.0001$ \\
\hline Diastolic BP (mmHg) & $67.6 \pm 7.6$ & $67.0 \pm 8.1$ & $67.6 \pm 7.9$ & 0.422 \\
\hline eGFR $\left(\mathrm{mL} / \mathrm{min} / 1.73 \mathrm{~m}^{2}\right)$ & $111.7 \pm 28.3$ & $104.6 \pm 25.6$ & $95.7 \pm 21.8$ & $<0.0001$ \\
\hline \multicolumn{5}{|c|}{$\begin{array}{l}\text { Data are expressed as mean } \pm S D, n(\%) \text {, median (interquartile range). } P \text { values are for trends across } \\
\text { tertiles. }\end{array}$} \\
\hline \multicolumn{5}{|c|}{$\begin{array}{l}\text { Abbreviations: } \mathrm{HbA}_{1 \mathrm{c}} \text { : glycosylated hemoglobin, } \mathrm{CSII} \text { : continuous subcutaneous insulin infusion. MDI: } \\
\text { multiple doses of insulin. IU/Kg/day: insulin dose pro Kg Day. TG/HDL ratio: triglycerides to HDL } \\
\text { ratio. TC/HDL ratio: Cholesterol to HDL ratio. SPISE: single-point insulin sensitivity estimator } \mathrm{N} / \mathrm{L} \\
\text { ratio: Neutrophil/Lymphocyte ratio. BP: blood pressure. eGFR: estimated glomerular filtration rate }\end{array}$} \\
\hline
\end{tabular}

When the analysis was repeated by sex, the same statistical differences (except for $\mathrm{HbA}_{1 \mathrm{c}}$ ) were observed in boys, whereas in girls a statistical difference between tertiles of UA was observed for age, daily insulin dose, presence of autoimmune diseases, HDL, TC/HDL ratio, N/L ratio and eGFR ( $p<0.05-0.0001)$ (Tables 3-4). The proportions (mean and $95 \% \mathrm{Cl}$ ) of youth with abnormal CMRFs across tertiles of UA both in boys and girls are shown in Figure 1. A statistically significant increase across UA tertiles was found in both sexes for high TC/HDL ratio, and MRGFR. In boys, higher prevalence of high blood pressure was found, whereas in the female group the prevalence of high N/L ratio varied across UA tertiles. 
Table 3

Anthropometric and biochemical features in boys according to sex-specific tertiles of uric acid.

\section{Uric acid tertiles}

\begin{tabular}{|c|c|c|c|c|}
\hline $\mathrm{UA}(\mathrm{mg} / \mathrm{dl})$ & $<3.0$ & $\geq 3.0<3.9$ & $\geq 3.9$ & \\
\hline$n=716$ & 216 & 254 & 246 & $p$ value \\
\hline Age (years) & $13.2 \pm 2.8$ & $13.8 \pm 3.1$ & $13.9 \pm 3.0$ & 0.028 \\
\hline $\mathrm{BMI}\left(\mathrm{kg} / \mathrm{m}^{2}\right)$ & $19.8 \pm 3.1$ & $20.9 \pm 3.9$ & $21.3 \pm 3.8$ & $<0.0001$ \\
\hline BMI-SDS & $-0.20 \pm 0.9$ & $0.02 \pm 1.0$ & $0.09 \pm 1.1$ & 0.004 \\
\hline Diabetes duration (years) & $6.3 \pm 3.2$ & $6.7 \pm 3.3$ & $6.6 \pm 3.2$ & 0.346 \\
\hline Autoimmune diseases & $46(21)$ & $55(22)$ & $55(22)$ & 0.961 \\
\hline Insulin dose (IU/Kg/day) & $0.82(0.66-1.06)$ & $0.84(0.62-1.16)$ & $0.95(0.72-1.18)$ & 0.022 \\
\hline $\mathrm{HbA}_{1 \mathrm{c}}(\%)$ & $7.7(7.2-8.4)$ & $7.6(7.0-8.4)$ & $7.6(7.0-8.4)$ & 0.308 \\
\hline $\mathrm{HbA}_{1 \mathrm{c}}(\mathrm{mmol} / \mathrm{mol})$ & $61.0(55.0-68.0)$ & $60.0(53.0-68.0)$ & $60.0(53.0-68.1)$ & 0.308 \\
\hline CSII, n (\%) & $75(35)$ & $93(37)$ & $77(31)$ & 0.449 \\
\hline Albuminuria, (\%) & $9(4.2)$ & $14(5.5)$ & $14(5.7)$ & 0.726 \\
\hline Total cholesterol (mg/dL) & $166.3 \pm 31.8$ & $168.1 \pm 31.9$ & $162.0 \pm 30.8$ & 0.089 \\
\hline $\mathrm{HDL}(\mathrm{mg} / \mathrm{dL})$ & $61.9 \pm 11.9$ & $59.9 \pm 12.8$ & $56.9 \pm 12.2$ & $<0.0001$ \\
\hline Triglycerides (mg/dL) & $55.0(43.0-68.0)$ & $60.0(46.0-75.0)$ & $61.0(47.0-84.0)$ & 0.001 \\
\hline TG/HDL ratio & $0.89(0.66-1.20)$ & $1.02(0.71-1.41)$ & $1.06(0.77-1.54)$ & $<0.0001$ \\
\hline $\mathrm{TC} / \mathrm{HDL}$ ratio $(\mathrm{mg} / \mathrm{dL})$ & $2.6(2.3-3.1)$ & $2.8(2.4-3.3)$ & $2.8(2.5-3.3)$ & 0.006 \\
\hline SPISE index & $11.1 \pm 2.7$ & $10.2 \pm 2.7$ & $9.7 \pm 2.6$ & $<0.0001$ \\
\hline $\mathrm{N} / \mathrm{L}$ ratio & $1.36(0.99-1.76)$ & $1.33(1.00-1.78)$ & $1.40(1.10-1.85)$ & 0.015 \\
\hline Systolic BP (mmHg) & $106.9 \pm 11.3$ & $109.0 \pm 12.2$ & $111.6 \pm 12.2$ & $<0.0001$ \\
\hline Diastolic BP (mmHg) & $66.9 \pm 7.5$ & $66.7 \pm 8.3$ & $68.2 \pm 8.2$ & 0.096 \\
\hline $\operatorname{eGFR}\left(\mathrm{mL} / \mathrm{min} / 1.73 \mathrm{~m}^{2}\right)$ & $115.1 \pm 27.4$ & $107.5 \pm 24.4$ & $96.9 \pm 23.1$ & $<0.0001$ \\
\hline
\end{tabular}

Data are expressed as mean \pm SD, $\mathrm{n}(\%)$, median (interquartile range). $P$ values are for trends across tertiles.

Abbreviations: $\mathrm{HbA}_{1 \mathrm{c}}$ : glycosylated hemoglobin, CSIl: continuous subcutaneous insulin infusion. MDI: multiple doses of insulin. IU/Kg/day: insulin dose pro Kg Day. TG/HDL ratio: triglycerides to $\mathrm{HDL}$ ratio. TC/HDL ratio: Cholesterol to HDL ratio. SPISE: single-point insulin sensitivity estimator N/L ratio: Neutrophil/Lymphocyte ratio. BP: blood pressure. eGFR: estimated glomerular filtration rate 
Table 4

Anthropometric and biochemical features in girls according to sex-specific tertiles of uric acid.

\section{Uric acid tertiles}

\begin{tabular}{|c|c|c|c|c|}
\hline $\mathrm{UA}(\mathrm{mg} / \mathrm{dl})$ & $<3.0$ & $\geq 3.0<3.8$ & $\geq 3.8$ & \\
\hline$n=607$ & 187 & 217 & 203 & $p$ value \\
\hline Age (years) & $13.0 \pm 3.3$ & $13.4 \pm 3.2$ & $13.8 \pm 3.0$ & 0.041 \\
\hline $\mathrm{BMI}\left(\mathrm{kg} / \mathrm{m}^{2}\right)$ & $20.8 \pm 3.8$ & $20.6 \pm 3.5$ & $21.0 \pm 3.9$ & 0.595 \\
\hline BMI-SDS & $0.19 \pm 1.0$ & $0.10 \pm 1.0$ & $0.11 \pm 1.0$ & 0.616 \\
\hline Diabetes duration (years) & $6.3 \pm 3.2$ & $6.5 \pm 3.3$ & $6.5 \pm 3.3$ & 0.787 \\
\hline Autoimmune diseases & $39(21)$ & $44(20)$ & $50(30)$ & 0.047 \\
\hline Insulin dose (IU/Kg/day) & $0.86(0.69-1.07)$ & $0.90(0.71-1.10)$ & $0.96(0.80-1.26)$ & 0.040 \\
\hline $\mathrm{HbA}_{1 \mathrm{c}}(\%)$ & $7.8(7.2-8.6)$ & $7.7(7.1-8.4)$ & $7.6(6.9-8.3)$ & 0.085 \\
\hline $\mathrm{HbA} 1 \mathrm{c}(\mathrm{mmol} / \mathrm{mol})$ & $61.7(55.0-70.0)$ & $60.1(54.0-68.7)$ & $60.0(52.0-67.0)$ & 0.085 \\
\hline CSII, n (\%) & $66(35)$ & $79(36)$ & $70(34)$ & 0.918 \\
\hline Albuminuria, (\%) & $16(8.6)$ & $20(9.2)$ & $17(8.4)$ & 0.949 \\
\hline Total cholesterol (mg/dL) & $166.0 \pm 32.2$ & $164.7 \pm 30.9$ & $162.4 \pm 30.1$ & 0.515 \\
\hline $\mathrm{HDL}(\mathrm{mg} / \mathrm{dL})$ & $62.1 \pm 12.9$ & $58.2 \pm 12.8$ & $57.5 \pm 12.8$ & 0.001 \\
\hline Triglycerides (mg/dL) & $58.0(46.0-73.0)$ & $59.0(47.0-75.0)$ & $58.0(47.0-85.0)$ & 0.226 \\
\hline TG/HDL ratio & $0.98(0.69-1.23)$ & $1.05(0.77-1.42)$ & $1.07(0.79-1.48)$ & 0.012 \\
\hline TC/HDL ratio $(\mathrm{mg} / \mathrm{dL})$ & $2.7(2.3-3.1)$ & $2.8(2.4-3.4)$ & $2.8(2.4-3.4)$ & 0.019 \\
\hline SPISE index & $10.3 \pm 2.7$ & $10.2 \pm 2.5$ & $10.0 \pm 2.8$ & 0.452 \\
\hline $\mathrm{N} / \mathrm{L}$ ratio & $1.27(0.96-1.67)$ & $1.35(1.02-1.72)$ & $1.40(1.05-2.00)$ & 0.031 \\
\hline Systolic BP (mmHg) & $107.5 \pm 11.9$ & $108.7 \pm 12.1$ & $109.4 \pm 10.2$ & 0.247 \\
\hline Diastolic BP (mmHg) & $68.4 \pm 7.8$ & $67.4 \pm 7.8$ & $67.0 \pm 7.4$ & 0.179 \\
\hline eGFR $\left(\mathrm{mL} / \mathrm{min} / 1.73 \mathrm{~m}^{2}\right)$ & $107.9 \pm 28.9$ & $101.1 \pm 26.5$ & $94.2 \pm 20.1$ & $<0.0001$ \\
\hline
\end{tabular}

Data are expressed as mean \pm SD, $\mathrm{n}(\%)$, median (interquartile range). $P$ values are for trends across tertiles.

Abbreviations: $\mathrm{HbA}_{1 \mathrm{c}}$ : glycosylated hemoglobin, CSIl: continuous subcutaneous insulin infusion. MDI: multiple doses of insulin. IU/Kg/day: insulin dose pro $\mathrm{Kg}$ Day. TG/HDL ratio: triglycerides to $\mathrm{HDL}$ ratio. Chol/HDL ratio: Cholesterol to HDL ratio. SPISE: single-point insulin sensitivity estimator N/L ratio: Neutrophil/Lymphocyte ratio. BP: blood pressure. eGFR: estimated glomerular filtration rate 
In the whole study population, the highest tertile of UA was associated with 1.6-3.7 fold increased risk of high TG/HDL, TC/HDL ratio, HTN, N/L ratio and MRGFR, compared to the lowest tertile in the overall sample (Table 5). Except for the high N/L ratio, the same CMR profile was observed in boys. In girls, the highest tertile of UA was associated with a double increased risk of high TC/HDL ratio and N/L ratio, and MRGFR (Table 5).

Table 5

Odds ratio $(95 \% \mathrm{Cl})$ of cardiometabolic risk factors according to tertiles of uric acid, in all sample, in boys and in girls.

\begin{tabular}{|c|c|c|c|}
\hline All individuals* & Tertile I & Tertile II & Tertile III \\
\hline High TG/HDL ratio & 1.00 & $1.70(1.21-2.38)^{0.002}$ & $2.04(1.45-2.87)^{0.0001}$ \\
\hline High TC/HDL ratio & 1.00 & $1.97(1.42-2.73)^{0.0001}$ & $2.10(1.50-2.93)^{0.0001}$ \\
\hline High BP & 1.00 & $0.95(0.64-1.43)$ & $1.60(1.10-2.35)^{0.015}$ \\
\hline High N/L ratio & 1.00 & $1.05(0.76-1.45)$ & $1.57(1.14-2.16)^{0.005}$ \\
\hline MRGFR & 1.00 & $1.58(1.17-2.13)^{0.003}$ & $2.69(2.00-3.64)^{0.0001}$ \\
\hline \multicolumn{4}{|l|}{ Boys** } \\
\hline High TG/HDL ratio & 1.00 & $1.83(1.14-2.92)^{0.012}$ & $2.17(1.36-3.47)^{0.001}$ \\
\hline High TC/HDL ratio & 1.00 & $1.62(1.04-2.52)^{0.033}$ & $1.67(1.07-2.62)^{0.025}$ \\
\hline High BP & 1.00 & $1.18(0.67-2.10)$ & $2.80(1.63-4.73)^{0.0001}$ \\
\hline MRGFR & 1.00 & $1.81(1.14-2.86)^{0.012}$ & $3.70(2.35-5.82)^{0.0001}$ \\
\hline \multicolumn{4}{|l|}{ Girls*** } \\
\hline High TC/HDL ratio & 1.00 & $2.15(1.34-3.46)^{0.002}$ & $2.18(1.34-3.55)^{0.002}$ \\
\hline High N/L ratio & 1.00 & $1.14(0.70-1.86)$ & $2.01(1.26-3.23)^{0.004}$ \\
\hline MRGFR & 1.00 & $1.43(0.95-2.15)$ & $2.08(1.37-3.16)^{0.001}$ \\
\hline \multicolumn{4}{|c|}{${ }^{*} p$ value adjusted for centers, age, insulin dose/Kg/Die, $\mathrm{HbA}_{1 \mathrm{c}}$. } \\
\hline \multicolumn{4}{|c|}{${ }^{\star \star} p$ value adjusted for centers, age, BMI-SDS, insulin dose/Kg/Die. } \\
\hline$\star \star \star \star p$ value adjustec & cer & e, insulin dose/Kg/ & \\
\hline
\end{tabular}

\section{Discussion}

Our study showed a close association between UA and CMRFs in children and adolescents with T1D. This association was remarkable and supports the role of UA as a potential early marker of 
cardiometabolic risk in this population. Furthermore, our study showed that the CMR risk profile associated with UA is worse in males than in females.

High levels of UA have been reported in adults with hypertension, obesity and metabolic syndrome [1]. Furthermore, prospective studies have shown the usefulness of UA in predicting incident cardio-renal events both in individuals with type 1 and type 2 diabetes [2-7].

In children, recent studies have shown a strong association between UA, nonalcoholic fatty liver disease mildly reduced eGFR and glucose dysmetabolism $[10,11]$. These studies have been performed in obese youths, whereas few and conflicting observations have been reported in youths with T1D [12, 13]. Indeed, Lytvyn et al. could not detect any relationship between UA and cardio-renal abnormalities in their study conducted in 180 adolescents with T1D [13]. In contrast, Słomiński et al. demonstrated a positive association between UA and both nephropathy and subclinical inflammatory marker concentrations in boys but not in girls with T1D [12].

In our study, we performed a comprehensive analysis including markers of low grade-inflammation and UA levels separately in the two sexes. To the best of our knowledge, our study demonstrates for the first time a robust association between CMRFs and UA in children and adolescents with T1D, which was more pronounced in boys than in girls.

Uric acid levels are higher in adult males than females, as well in obese boys than girls $[1,10,11]$. This difference is likely due to a distinct roles of sex hormones and a higher muscle mass in males [17]. In our study population, we did not observe any difference in UA levels between the two sexes, probably for the absence of a significant difference in the value of BMI-SDS between the two sexes.

In childhood, high values of UA are influenced by ethnicity, age and sex, so a universal definition of abnormal UA levels is lacking. In non-obese youths values above 6 or $7 \mathrm{mg} / \mathrm{dL}$ in boys and 5 or $6 \mathrm{mg} / \mathrm{dL}$ in girls were previously been considered "elevated" $[18,19]$.

In children and adolescents with T1D levels of UA are lower than healthy individuals [20]. This result could lead to undermining the usefulness of assessing UA in clinical practice in youth with T1D. Despite this, our study showed a robust negative association between 'high normal' levels of UA and eGFR, in line with our previous studies [21,22] and extends a positive association between UA and different CMRFs in young people with T1D. Interestingly, the results differed by sex, with boys having an increased risk for high TG/HDL, TC/HDL ratio, high BP and MRGFR, whereas girls showed a high risk of abnormal TC/HDL ratio, N/L ratio and MRGFR.

The different impact of UA in males vs females is still undefined. In our sample, we confirm in boys an association between high BP and high levels of UA as reported in obese youths [23]. This suggests that levels of UA in the "high normal" range may identify boys with T1D at high risk of hypertension. This association may be mediated by low insulin sensitivity as demonstrated by an inverse relationship 
between the SPISE index, a validate index of low insulin-sensitivity, and BP across the sex-specific tertiles of UA.

In the present study, an association between the TG/HDL ratio and UA in boys was also detected. Insulin resistance may represent the link between the TG/HDL ratio and UA. Supporting our hypothesis is evident of a mutual increase in the TG/HDL ratio and a reduction of SPISE index in boys, but not in girls. Since HOMA-IR is inapplicable in patients with T1D, in the present study we used the SPISE index [24], to analyze the relationship between UA and insulin sensitivity.

A further novel finding from our study is the close association between high levels of UA and high TC/HDL ratio. This lipid ratio is considered a marker of atherogenic dyslipidemia [25] and a more sensitive predictor of cardiovascular events in adults than Total Cholesterol [26]. Interestingly, the association between UA and high TC/HDL ratio is shared by the two sexes, independently of BMI. This further supports the usefulness of UA as marker of early atherosclerosis in both sexes.

In adults and obese children, the relationship between UA and MRGFR is well established [1, 10], whereas this association has been little explored in T1D, especially in young people [12, 13]. The strong associations we found between UA and MRGFR in both boys and girls is in line with our previous reports $[21,22]$. The strength of this association sustains the evaluation of UA in clinical routine to identify youths with T1D at risk of decline of eGFR in both sexes.

Of particular interest is the link between a high $\mathrm{N} / \mathrm{L}$ ratio, as surrogate of low-grade inflammation, and high levels of UA in girls. The $N / L$ ratio has been recently studied in several conditions, such as cardiac, vascular, and kidney disease where the low-grade inflammation was potentially involved [27]. In particular, recent studies demonstrated that a higher N/L ratio represent a useful marker to identify diabetic kidney disease [28]. In our sample we observed a robust association between N/L ratio and high levels of UA in girls, but not in boys. This association may be supported by higher percentage of girls with concomitant autoimmune diseases as compared to boys. This finding contrasts with the findings of Słomiński et al. [12] describing a positive association between UA and subclinical inflammatory marker concentrations only in boys but not in girls. The reasons for this discrepancy are unknown and warrant further study.

Some limitations of the present study need to be acknowledged. Firstly, being a retrospective multicenter, clinic-based study, data were collected and analyzed across different centers. However, all laboratories were standardized and used similar methods that were previously aligned as part of previous study [29] although this can be a limitation. However, it is important to acknowledge that the study methods reflect real-word data collection and therefore closely reflect what can be implemented in daily clinical practice. The study population lacked diversity in terms of ethnicity, and this may limit the generalization of the study findings. However, despite these limitations, the present study, based on a large sample size and data collection, highlighted an important role of UA as potential early marker of cardiometabolic risk. 
In conclusion, our study demonstrates that measuring UA in youth with T1D could be useful to identify those at higher cardio-metabolic risk, although sex-related differences need to be taken into account. If

confirmed by future studies, the present findings could lead towards the implementation of UA as part of the set of investigations required in all youth with T1D from early stages of the disease to support prediction and prevention of cardio-metabolic complications.

\section{Declarations}

Ethics approval and consent to participate: the present study was approved by the internal review board of the ethic committee University "Federico II" of Naples (protocol number 55/20) and by the local ethics committees of the following participating centers: A.O.U. Salesi Hospital of Ancona, Giovanni XXIII Children's Hospital of Bari, University of Campania Luigi Vanvitelli, University Hospital of Parma, University Hospital of Verona, S. Orsola-Malpighi University Hospital of Bologna, A.O. Brotzu of Cagliari, Bambino Gesù Children's Hospital of Rome. Informed consent has been obtained from all parents and/or patients. This study was conducted in accordance with the Declaration of Helsinki. The clinical and biochemical parameters were anonymously entered in a database using an alphanumeric and progressive code.

Acknowledgements: we kindly thank the patients and their families who participated in the study

Authors' contributions: P.D.B. and E.M. wrote the manuscript. MLM suggested statistical analysis and P.D.B. oversaw the statistical analysis. E.M. and F.M.R. edited the manuscript. P.D.B., M.L.M., A.F., E.M. reviewed the manuscript. E.M., FMR, VC, MD, FDC, DI, BI, CM, GM, CR, EP, CAP, MRR, RS, TS collected data. E.M. and P.D.B. are the guarantors of this work and, as such, had full access to all the data in the study and take responsibility for the integrity of the data and the accuracy of the data analysis. All authors were responsible for drafting the article and revising it critically for important intellectual content. All authors approved the version to be published.

Data availability: the datasets generated and/or analysed during the current study are not publicly available due privacy/ethical restrictions but are available from the corresponding author (mozzilloenza@gmail.com) on reasonable request.

Competing Interests: the authors declare that they have no known competing financial interests or personal relationships that could have appeared to influence the work reported in this paper.

Funding statement this research did not receive any specific grant from funding agencies in the public, commercial, or not-for-profit sectors.

\section{References}

1. Sharaf El Din, U.A.A., Salem, M.M., Abdulazim, D.O. Uric acid in the pathogenesis of metabolic, renal, and cardiovascular diseases: a review. J Adv Res 8:537-548. doi: 10.1016/j.jare.2016.11.004 (2017) 
2. Rodrigues, T.C. et al. Serum uric acid predicts progression of subclinical coronary atherosclerosis in individuals without renal disease Diabetes Care 33:2471-3. doi: 10.2337/dc10-1007 (2010).

3. Cang, Y. et al. Serum uric acid revealed a U-shaped relationship with all-cause mortality and cardiovascular mortality in high atherosclerosis risk patients: the ASSURE Study. Front Cardiovasc Med 8:641513. doi: 10.3389/fcvm.2021.641513 (2021).

4. Zoppini, G. et al. Serum uric acid levels and incident chronic kidney disease in patients with type 2 diabetes and preserved kidney function. Diabetes Care 35:99-104. doi: 10.2337/dc11-1346 (2012).

5. Pilemann-Lyberg, S. et al. Uric acid is an independent risk factor for decline in kidney function, cardiovascular events, and mortality in patients with type 1 diabetes. Diabetes Care 42:1088-1094. doi: 10.2337/dc18-2173. (2019).

6. Bjornstad, P. et al. Serum uric acid predicts vascular complications in adults with type 1 diabetes: the coronary artery calcification in type 1 diabetes study. Acta Diabetol 51:783-91. doi: 10.1007/s00592-014-0611-1 (2014).

7. Ficociello, L.H. et al. High-normal serum uric acid increases risk of early progressive renal function loss in type 1 diabetes. Diabetes Care 33:1337-1343. doi: 10.2337/dc10-0227 (2010).

8. Doehner, W., Jankowska, E.A., Springer, J., Lainscak, M., Anker, S.D. Uric acid and xanthine oxidase in heart failure - emerging data and therapeutic implications. Int J Cardiol;15:15-9 (2016).

9. Moulin-Mares, S.R.A., Polyana, R., Oliosa. E.R., Zago-Gomes, M.P., Mill, J.G. Association of uric acid with cardiovascular risk in Brazilian children and adolescents. Nutr Metab Cardiovasc Dis 31:314321. doi: 10.1016/j.numecd.2020.09.012 (2021).

10. Di Bonito, P. et al. High uric acid, reduced glomerular filtration rate and non-alcoholic fatty liver in young people with obesity. J Endocrinol Invest 43:461-468. doi: 10.1007/s40618-019-01130-6 (2020).

11. Di Bonito, P. et al. Uric acid, impaired fasting glucose and impaired glucose tolerance in youth with overweight and obesity. Nutr Metab Cardiovasc Dis 31:675-680. doi: 10.1016/j.numecd.2020.10.007. (2021).

12. Słomiński, B., Skrzypkowska, M., Ryba-Stanisławowska, M., Brandtet, A. Sex-related association of serum uric acid with inflammation, kidney function and blood pressure in type 1 diabetic patients. Pediatr Diabetes 19:1014-1019. doi: 10.1111/pedi.12670 (2018).

13. Lytvyn, Y. et al. Association Between Plasma Uric Acid Levels and Cardiorenal Function in Adolescents with Type 1 Diabetes. Diabetes Care 39:611-616. doi: 10.2337/dc15-2345 (2016).

14. De Ferranti, S.D. et al. Cardiovascular risk reduction in high-risk pediatric patients: a scientific statement from the American Heart Association. Circulation 139:e603-e634. doi: 10.1161/CIR.0000000000000618 (2019).

15. Niechciał, E., Marcovecchio, M.L. Treatment of cardiometabolic risk factors in patients with type 1 diabetes. Curr Opin Pediatr 32:589-594. doi: 10.1097/MOP.0000000000000915 (2020).

16. Fornari, E. et al. Cardiovascular risk factors in children and adolescents with type 1 diabetes in Italy: a multicentric observational study. Pediatr Diabetes 21:1546-1555. doi: 10.1111/pedi.13123 (2020). 
17. Alvim, R.O., Siqueira, J.H., Zaniqueli, D., Dutra, D.M., Oliosa, P.R., Mill, J.G. Influence of muscle mass on the serum uric acid levels in children and adolescents. Nutr Metab Cardiovasc Dis;10;30:300-305. doi: 10.1016/j.numecd.2019.08.019 (2020).

18. Moulin-Mares, S.R.A. et al. Uric acid reference values: report on 1750 healthy Brazilian children and adolescents. Pediatr Res;89:1855-1860 (2021).

19. Dai, C. et al. Age and Gender-Specific Reference Intervals for Uric Acid Level in Children Aged 5-14 Years in Southeast Zhejiang Province of China: Hyperuricemia in Children May Need Redefinition. Front Pediatr;10;9:560720. doi: 10.3389/fped.2021.560720 (2021).

20. Lytvyn, Y., Škrti, M., Yang, G.K., Yip, P.M., Perkins, B.A., Cherney, D.Z.I. Glycosuria-mediated urinary uric acid excretion in patients with uncomplicated type 1 diabetes mellitus. Am J Physiol Renal Physiol 308:F77-F83. doi: 10.1152/ajprenal.00555.2014 (2015).

21. Di Bonito, P. et al. "The DIAbetic kidney disease in youth with type 1 diabetes in ITALY" (DIANITALY) Study Group. Albuminuric and non-albuminuric reduced eGFR phenotypes in youth with type 1 diabetes: Factors associated with cardiometabolic risk. Nutr Metab Cardiovasc Dis 31:2033-2041. doi: 10.1016/j.numecd.2021.03.019 (2021).

22. Di Bonito, P. et al. Non-albuminuric reduced eGFR phenotype in children and adolescents with type 1 diabetes. Diabetes Res Clin Pract 155:107781. doi: 10.1016/j.diabres.2019.07.005 (2019).

23. Lurbe, E., Torro, M.I., Alvarez-Pitti, J., Redon, J., Borghi, C., Redon, P. Uric acid is linked to cardiometabolic risk factors in overweight and obese youths. J Hypertens;36:1840-1846. doi: 10.1097/HJH.0000000000001814 (2018).

24. Correa-Burrows, P., Blanco, E., Gahagan, S., Burrows, R. Validity assessment of the single-point insulin sensitivity estimator (SPISE) for diagnosis of cardiometabolic risk in post-pubertal Hispanic adolescents. Sci Rep;10:14399. doi: 10.1038/s41598-020-71074-y (2020).

25. Millán, J. et al. Lipoprotein ratios: Physiological significance and clinical usefulness in cardiovascular prevention. Vasc Health Risk Manag;5:757-65 (2009)

26. Genest, J., Frohlich, J., Fodor, G., McPherson, R. The Working Group on Hypercholesterolemia and Other Dyslipidemias. Recommendations for the management of dyslipidemia and the prevention of cardiovascular disease: 2003 update. CMAJ;169:921-924 (2003).

27. Bhat, T. et al. Neutrophil to lymphocyte ratio and cardiovascular diseases: a review. Expert Rev Cardiovasc Ther;11:55-9. doi: 10.1586/erc.12.159 (2013).

28. Khandare, S.A., Chittawar, S., Nahar, N., Dubey, T.N., Qureshi, Z.. Study of Neutrophil-lymphocyte Ratio as Novel Marker for Diabetic Nephropathy in Type 2 Diabetes. Indian J Endocrinol Metab;21(3):387392. doi: 10.4103/ijem.IJEM_476_16 (2017).

29. Montano, D. Certifying leaders? high-quality management practices and healthy organizations: an ISO-9000 based standardization approach. Ind Health 54:324-336. doi: 10.2486/indhealth.20150178 (2016).

30. Cacciari, E. et al. Italian cross-sectional growth charts for height, weight and BMI (2 to $20 \mathrm{yr}$ ). J Endocrinol Invest 29:581-93. doi: 10.1007/BF03344156 (2006). 
31. De Souza, V.C. et al. Schwartz formula: is one k-coefficient adequate for all children? PLoS One;7:e53439 (2021).

32. Levey, A.S. et al. Nomenclature for kidney function and disease: report of a kidney disease: improving global outcomes (KDIGO) consensus conference. Kidney Int 97:1117e29. doi: 10.1016/j.kint.2020.02.010 (2020).

33. Lurbe, E. et al. European Society of Hypertension guidelines for the management of high blood pressure in children and adolescents. J Hypertens; 34: 1887-1920 (2016).

34. Donaghue, K.C. et al. ISPAD Clinical Practice Consensus Guidelines 2018: Microvascular and macrovascular complications in children and adolescents. Pediatr Diabetes19 Suppl 27:262-274. doi: 10.1111/j.1399-5448.2009.00576.x (2018).

35. Flynn, J.T. et al. Clinical practice guideline for screening and management of high blood pressure in children and adolescents. Pediatrics; 140: e20171904 (2017).

\section{Figures}

Figure 1. Proportions (mean and $95 \% \mathrm{CI}$ ) of youth with abnormal CMRFs across tertiles of UA both in boys and girls.
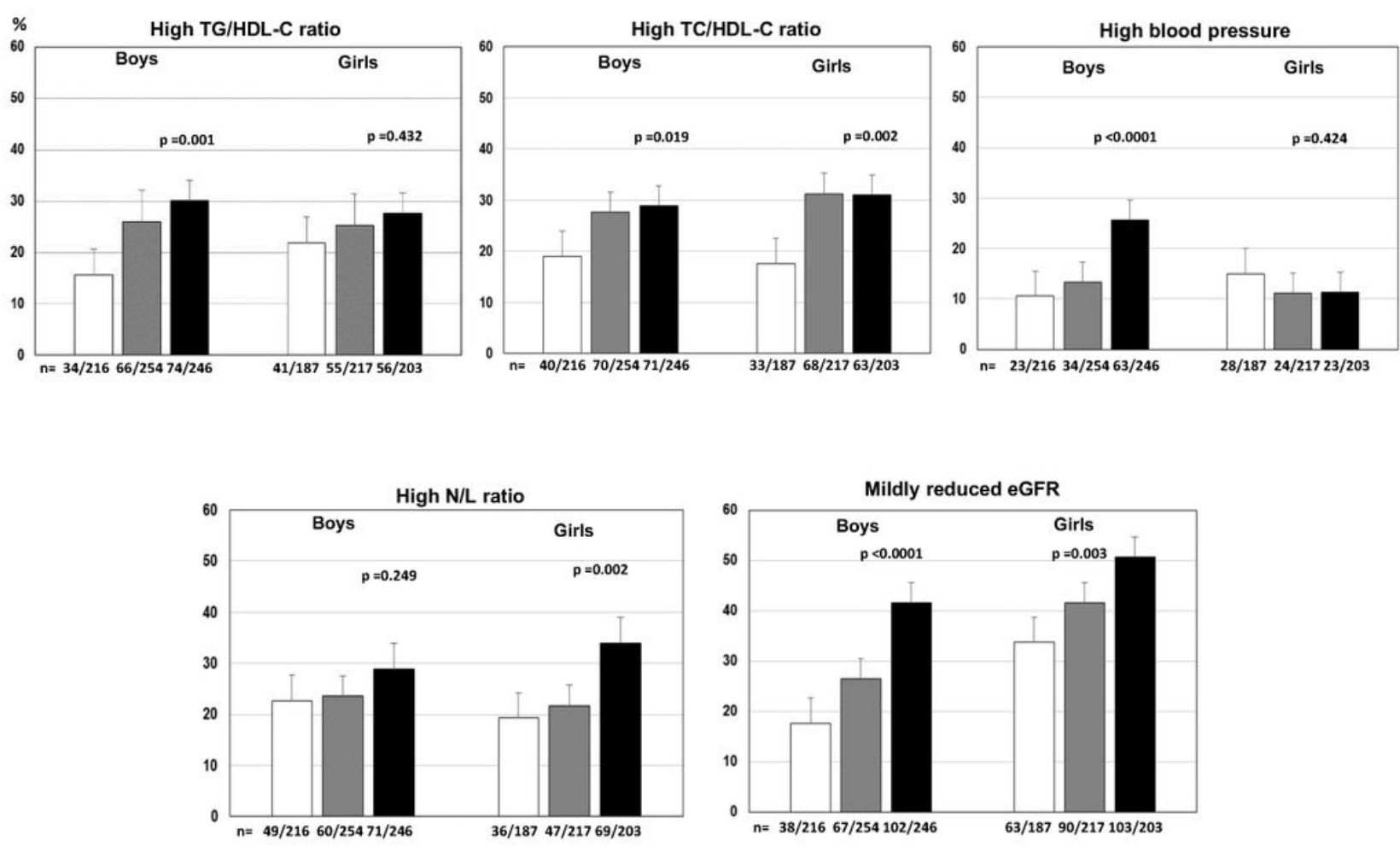

Figure 1 
See image above for figure legend

Page 19/19 